\title{
Singular Vectors of the Virasoro Algebra in Terms of Jack Symmetric Polynomials
}

\author{
Katsuhisa Mimachi, Yasuhiko Yamada \\ Department of Mathematics, Kyushu University, Hakozaki, Fukuoka 812, Japan
}

Received: 25 November 1994 / in revised form: 6 March 1995

\begin{abstract}
We present an explicit formula of the Virasoro singular vectors in terms of Jack symmetric polynomials. The parameter $t$ in the Virasoro central charge $c=13-6(t+1 / t)$ is just identified with the deformation parameter $\alpha$ of Jack symmetric polynomials $J_{\lambda}(\alpha)$. As a by-product, we obtain an integral representation of Jack symmetric polynomials indexed by the rectangular Young diagrams.
\end{abstract}

\section{Introduction}

The Virasoro algebra is an infinite dimensional Lie algebra defined by the generators $l_{m}(m \in \mathbb{Z})$ and $c$ with the relations

$$
\left[l_{n}, l_{m}\right]=(n-m) l_{m+n}+\frac{c}{12}\left(n^{3}-n\right) \delta_{m+n, 0}
$$

for $m, n \in \mathbb{Z}$ and $\left[c, l_{m}\right]=0$ for $m \in \mathbb{Z}$. Among many mathematicians and physicists to investigate the representation theory of the Virasoro algebra (see $[2,3]$ for instance), Tsuchiya and Kanie classified the singular vectors of the Fock space representations in [8], where they constructed the integral representation of the singular vectors. But they missed obtaining explicit forms of such singular vectors. The present article will be devoted to this point: Namely, we express the singular vectors in terms of some symmetric polynomials, called Jack symmetric polynomials.

Jack symmetric polynomials were first introduced by Jack in 1970 to evaluate some definite integral. Subsequently, the systematic study was begun by Macdonald, who considered even more general cases: Macdonald's symmetric polynomials [6].

In this paper we show that the singular vectors of the Virasoro algebra are given by Jack symmetric polynomials indexed by the rectangular Young diagrams.

We believe that such realization of Jack symmetric polynomials would be helpful in future research both of the representation theory of infinite dimensional algebras and of hypergeometric type functions in several variables.

Some basic notations about the symmetric polynomials, with definition of Jack symmetric polynomials, are recalled in Sect. 2. We give the main result in Sect. 3 , 
after a short review on the Fock space representation. Sections 4 and 5 are devoted to its proof. The key ingredient of our proof is an expression of Jack symmetric polynomials by Euler-Selberg type integrals.

We remark that the present result can be viewed as a generalization of the result in [9]. They showed, by a direct computation, that $c=1$ singular vectors are given by the Schur polynomials.

Among the related works we refer the reader to [1], where the singular vectors in Verma modules and associated differential equations were studied.

\section{Jack Symmetric Polynomials}

We recall the basic notion about the symmetric polynomials by following the standard reference [4].

A partition $\lambda$ is a sequence $\lambda=\left(\lambda_{1}, \lambda_{2}, \ldots, \lambda_{n}\right)$ of non-negative integers in decreasing order; $\lambda_{1} \geqq \lambda_{2} \geqq \cdots \geqq \lambda_{n} \geqq 0$. The number of the non-zero $\lambda_{i}$ 's is called the length of $\lambda$, denoted by $l(\lambda)$; and the sum of $\lambda_{l}$ 's is the weight of $\lambda$, denoted by $|\lambda|$. Given a partition $\lambda=\left(\lambda_{1}, \lambda_{2}, \ldots, \lambda_{n}\right)$, we define the conjugate partition $\lambda^{\prime}=\left(\lambda_{1}^{\prime}, \lambda_{2}^{\prime}, \ldots, \lambda_{n}^{\prime}\right)$ by $\lambda_{l}^{\prime}=\operatorname{Card}\left\{j ; \lambda_{j} \geqq i\right\}$. The dominace (or natural) ordering on partitions is defined as $\lambda \geqq \mu \Leftrightarrow|\lambda|=|\mu|$ and $\lambda_{1}+\cdots+\lambda_{i} \geqq \mu_{1}+\cdots+$ $\mu_{t}$ for all $i \geqq 1$.

Sometimes, given a partition $\lambda$ we also use a notation which indicates the number of times each integer occurs: $\lambda=\left(1^{m_{1}}, 2^{m_{2}}, \ldots\right)$ with $m_{i}=m_{i}(\lambda)=\operatorname{Card}\left\{j ; \lambda_{j}=i\right\}$.

For $\beta=\left(\beta_{1}, \ldots, \beta_{n}\right) \in \mathbb{Z}^{n}$ we denote by $x^{\beta}$ the monomial $x^{\beta}=x_{1}^{\beta_{1}} \ldots x_{n}^{\beta_{n}}$. If $\lambda$ is a partition of length $l(\lambda) \leqq n$, the monomial symmetric polynomial $m_{\lambda}$ is defined by $m_{\lambda}\left(x_{1}, \ldots, x_{n}\right)=\sum x^{\beta}$, where the sum is taken over all distinct permutations $\beta$ of $\lambda=\left(\lambda_{1}, \lambda_{2}, \ldots, \lambda_{n}\right)$. For each integer $r \geqq 0$, the $r^{\text {th }}$ elementary symmetric polynomial $e_{r}$ and the $r^{\text {th }}$ power sum $p_{r}$ are defined by $e_{r}=\sum_{1 \leqq i_{1}<i_{2}<\cdots<i_{r} \leqq n} x_{t_{1}} x_{t_{2}} \cdots x_{l_{r}}=$ $m_{\left(1^{\prime}\right)}$ and $p_{r}=\sum_{l=1}^{n} x_{i}^{r}=m_{(r)}$, respectively.

Jack symmetric polynomials $J_{\lambda}=J_{\lambda}\left(x_{1}, \ldots, x_{n} ; \alpha\right)$, indexed by partitions $\lambda=$ $\left(\lambda_{1}, \lambda_{2}, \ldots, \lambda_{n}\right)$ of length $l(\lambda) \leqq n$, are defined as the eigenfunctions for the operator

$$
D(\alpha)=\frac{\alpha}{2} \sum_{l=1}^{n} x_{i}^{2} \frac{\partial^{2}}{\partial x_{i}^{2}}+\sum_{1 \leqq i \neq j \leqq n} \frac{x_{l}^{2}}{x_{i}-x_{j}} \frac{\partial}{\partial x_{i}} .
$$

Namely, for a fixed partition $\lambda, J_{\lambda}$ is a unique solution of

$$
D(\alpha) J_{\lambda}=e_{\lambda}(\alpha) J_{\lambda}
$$

and

$$
e_{\lambda}(\alpha)=\frac{\alpha}{2} \sum_{i=1}^{n} \lambda_{l}\left(\lambda_{i}-1\right)-\sum_{i=1}^{n}(i-1) \lambda_{i}+(n-1)|\lambda|
$$

in the form

$$
J_{\lambda}=m_{\lambda}+\sum_{\mu<\lambda} a_{\lambda \mu} m_{\mu}
$$

for $a_{\lambda \mu} \in \mathbb{C}(\alpha)$. 
Jack symmetric polynomials $J_{\lambda}$ are a common generalization of some wellknown polynomials. Indeed, for particular cases of $\alpha$, polynomials $J_{\lambda}$ reduce to the following:

(i) When $\alpha=1$, they are the Schur polynomials $s_{\lambda}\left(x_{1}, \ldots, x_{n}\right)$, i.e. the characters of the polynomial representations of $G L(n ; \mathbb{C})$.

(ii) When $\alpha=2$, they are the zonal polynomials $Z_{\lambda}\left(x_{1}, \ldots, x_{n}\right)$, i.e. the zonal spherical functions on $G L(n) / S O(n)$.

(iii) When $\alpha=1 / 2$, they are the zonal spherical functions on $G L(2 n) / S p(n)$.

(iv) When $\alpha=0$, we have $J_{\lambda}\left(x_{1}, \ldots, x_{n} ; 0\right)=e_{\lambda^{\prime}}\left(x_{1}, \ldots, x_{n}\right)$, the product of elementary symmetric polynomials corresponding to the conjugate $\lambda^{\prime}$ of $\lambda$.

(v) If $\alpha$ tends to $\infty$, suitably normalized $J_{\lambda}\left(x_{1}, \ldots, x_{n} ; \alpha\right)$ reduce to the monomial symmetric polynomials $m_{\lambda}\left(x_{1}, \ldots, x_{n}\right)$.

For details of Jack symmetric polynomials, we refer the reader to [5 and 7].

\section{Fock Representations and Main Theorem}

To state our main result, we prepare the notion of the Fock space representations of the Virasoro algebra.

First set

$$
\alpha_{0}=\frac{\beta}{2}-\frac{1}{\beta}, \quad \alpha_{r, s}=(1+r) \frac{\beta}{2}-(1+s) \frac{1}{\beta} \quad \text { and } \quad t=\frac{\beta^{2}}{2}
$$

for $\beta \in \mathbb{C}$ and $r, s \in \mathbb{Z}$.

The Heisenberg algebra $\mathscr{A}$ is a $\mathbb{C}$-algebra generated by $a_{n}(n \in \mathbb{Z})$ with the relations

$$
\left[a_{n}, a_{m}\right]=n \delta_{n+m, 0} .
$$

Denote by $F_{\alpha}$ a left $\mathscr{A}$-module defined by the generator $|\alpha\rangle$ and the relations

$$
a_{n}|\alpha\rangle=0(n>0), \quad a_{0}|\alpha\rangle=\alpha|\alpha\rangle \text {. }
$$

When we put

$$
L_{n}=\frac{1}{2} \sum_{m \in \mathbf{Z}}: a_{n-m} a_{m}:-\alpha_{0}(n+1) a_{n}
$$

for $n \in \mathbb{Z}$, we obtain the relations

$$
\left[L_{n}, a_{m}\right]=-m a_{n+m}-\alpha_{0} n(n+1) \delta_{n+m, 0} \quad(m, n \in \mathbb{Z})
$$

and

$$
\left[L_{n}, L_{m}\right]=(n-m) L_{n+m}+\frac{c}{12}\left(n^{3}-n\right) \delta_{n+m, 0} \quad(m, n \in \mathbb{Z}),
$$

the Virasoro algebra with the central charge $c=1-12 \alpha_{0}^{2}=13-6\left(t+\frac{1}{t}\right)$. Here we use the normal products defined by

$$
: a_{n} a_{m}:= \begin{cases}a_{n} a_{m} & (n \leqq m), \\ a_{m} a_{n} & (n>m)\end{cases}
$$


Moreover, on the module $F_{\alpha}$ for each $\alpha \in \mathbb{C}$, we have the relations

$$
L_{n}|\alpha\rangle=0\left(n \in \mathbf{Z}_{>0}\right) \text { and } L_{0}|\alpha\rangle=h_{\alpha}|\alpha\rangle
$$

with $h_{\alpha}=\frac{\alpha^{2}}{2}-\alpha_{0} \alpha$. These imply that $F_{\alpha}$ can be considered as the highest weight module of the Virasoro algebra with the highest weight vector $|\alpha\rangle$ and the highest weight $h_{\alpha}$. We call this module $F_{\alpha}$ the Fock space representation of the Virasoro algebra.

An element $|\chi\rangle \in F_{\alpha}$ satisfying the relations

$$
L_{n}|\chi\rangle=0\left(n \in \mathbf{Z}_{>0}\right) \quad \text { and } \quad L_{0}|\chi\rangle=\left(h_{\alpha}+N\right)|\chi\rangle
$$

is called the singular vector of degree $N \in \mathbb{Z}$. To analyze the structure of the Fock module $F_{\alpha}$, it plays an important role. Denote by $S F_{\alpha}(N)$ the set of singular vectors of degree $N$ in $F_{\alpha}$. If $\alpha \notin\left\{\alpha_{r, s} ; r, s \in \mathbb{Z}_{>0}\right.$ or $\left.r, s \in \mathbb{Z}_{<0}\right\}, F_{\alpha}$ is irreducible and $\operatorname{dim} S F_{\alpha}(N)=0$ for any $N$. If $\alpha=\alpha_{r, s}$ for $r, s \in \mathbb{Z}_{>0}, \operatorname{dim} S F_{\alpha}(r s)=1$, i.e. there exists a unique singular vector $\left|\chi_{r, s}\right\rangle \in F_{\alpha_{r, s}}$ of degree $N=r s$ up to a constant factor. Furthermore, there exists a polynomial $P_{r, s} \in \mathbb{C}\left[a_{-1}, a_{-2}, a_{-3}, \ldots, a_{-r s}\right]$ whose coefficients are rational functions in $\beta$ such that $S F_{\alpha_{r, s}}(r s)=\mathbb{C} P_{r, s}\left|\alpha_{r, s}\right\rangle$. Since we are not interested in the constant factor for defining the polynomial $P_{r, s}$, we fix it in what follows by the formula (4) below.

Here we reach the stage to give our main result:

Theorem 1. We have

$$
P_{r, s}=\rho_{n}\left(J_{\left(r^{s}\right)}\left(x_{1}, \ldots, x_{n} ; t\right)\right)
$$

where $n \geqq r s$ and

$$
\rho_{n}: \mathbb{C}\left[x_{1}, x_{2}, \ldots, x_{n}\right]^{S_{n}} \rightarrow \mathbb{C}\left[a_{-1}, a_{-2}, a_{-3}, \ldots, a_{-n}\right]
$$

is an algebra homomorphism determined by

$$
\rho_{n}\left(p_{m}(x)\right)=(-1)^{m-1} \beta a_{-m}
$$

for $1 \leqq m \leqq n$.

\section{Proof of Theorem}

On the manifold $M_{r}=\left\{\left(z_{1}, \ldots, z_{r}\right) \in\left(\mathbb{C}^{*}\right)^{r} ; z_{l} \neq z_{j}(1 \leqq i<j \leqq r)\right\}$, set

$$
\Phi=\prod_{1 \leqq i<j \leqq r}\left(z_{i}-z_{j}\right)^{2 t} \prod_{1 \leqq i \leqq r} z_{i}^{(1-r) t-1}
$$

and $d \zeta=d z_{1} \ldots d z_{r}$. Let $H_{r}\left(M_{r} ; S\right)$ be the twisted de Rham homology with coefficient $S$, local system associated with $\Phi$. This homology is studied in [8]. They construct a cycle $\mathscr{C} \in H_{r}\left(M_{r} ; S\right)$ which gives the following.

Lemma 2. There exists a cycle $\mathscr{C} \in H_{r}\left(M_{r} ; S\right)$ such that

(a) unless $m_{1}+\cdots+m_{r}=0$,

$$
\int_{\mathscr{C}} \Phi \prod_{1 \leqq l \leqq r} z_{i}^{m_{i}} d \zeta=0 \quad \text { for } \quad m_{i} \in \mathbb{Z}
$$


(b) if $d(d+1) t \notin \mathbb{Z}$ and $d(r-d) t \notin \mathbb{Z}$ for $1 \leqq d \leqq r-1$,

$$
\int_{\mathscr{C}} \Phi d \zeta=\frac{1}{(r-1) !} \prod_{j=1}^{r-1} \frac{\Gamma((j-r) t) \Gamma((j+1) t+1))}{\Gamma(t+1)} .
$$

See Proposition 4.2 in [8].

It is known that a polynomial given by the integral

$$
P_{r, s}=\int_{\mathscr{C}} \prod_{1 \leqq l<J \leqq r}\left(z_{l}-z_{J}\right)^{2 t} \prod_{i=1}^{r} z_{l}^{(1-r) t-1-s} \widehat{E}\left(z_{i}\right) d \zeta
$$

on the above cycle $\mathscr{C}$ determines the singular vector $\left|\chi_{r, s}\right\rangle=P_{r, s}\left|\alpha_{r, s}\right\rangle$.

Here $\widehat{E}(z)$ is defined by

$$
\widehat{E}(z)=\exp \left(\beta \sum_{m=1}^{n} \frac{a_{-m}}{m} z^{m}\right)
$$

with $n \geqq r s$. Since $\widehat{E}(z)=\rho_{n}(E(z))$ with $E(z)=\sum_{l=0}^{n} e_{i}(x) z^{i}=\prod_{k=1}^{n}\left(1+x_{k} z\right)$, the following is enough to prove our theorem:

Proposition 3. Jack symmetric polynomial $J_{\left(r^{s}\right)}\left(x_{1}, \ldots, x_{n} ; t\right)$ for $r \leqq n$ is expressed by

$$
N_{r}(t) \int_{\mathscr{C}} \Phi \prod_{1 \leqq i \leqq r} z_{l}^{-s} E\left(z_{i}\right) d \zeta
$$

where

$$
N_{r}(t)=(r-1) ! \prod_{j=1}^{r-1} \frac{\Gamma(t+1)}{\Gamma((j-r) t) \Gamma((j+1) t+1))} .
$$

Proof. By Lemma 2, we see that the integral expresses a homogeneous polynomial of degree $r s$ and that $m_{\left(r^{s}\right)}$ is its leading term. Hence we need only to prove that the integral

$$
\int_{\mathscr{C}} \Phi \prod_{1 \leqq l \leqq r} z_{i}^{-s} E\left(z_{i}\right) d \zeta
$$

satisfies the differential equation (2) with $\alpha=t$, which will be given in the next section $^{1}$

\section{The Differential Equation}

In this section, we denote by

$$
\phi_{1} \equiv \phi_{2}
$$

the equality

$$
\int_{\mathscr{C}} \phi_{1} \tilde{\Phi} d \zeta=\int_{\mathscr{C}} \phi_{2} \tilde{\Phi} d \zeta
$$

1 During our preparation of the present paper, we received the preprint "Collective Field Theory, Calogero-Sutherland Model and Generalized Matrix Models" by H. Awata et al. (hep-th/9411053). Although this kind of integral representation for the Jack polynomials is also considered there, the argument on the cycles is missing. 
with

$$
\tilde{\Phi}=\Phi \prod_{1 \leqq i \leqq r} z_{i}^{-s} E\left(z_{i}\right)=\prod_{1 \leqq i<j \leqq r}\left(z_{l}-z_{j}\right)^{2 t} \prod_{1 \leqq i \leqq r} z_{i}^{(1-r) t-1-s} \prod_{\substack{1 \leqq l \leqq r \\ 1 \leqq k \leqq n}}\left(1+x_{k} z_{i}\right)
$$

for our fixed cycle $\mathscr{C}$.

Lemma 4. We get

$$
\sum_{\substack{1 \leqq k \leqq n \\ 1 \leqq l \leqq r}} \frac{1}{1+x_{k} z_{i}} \equiv r(n-s)
$$

and

$$
\begin{aligned}
& \{(1-r) t+n-s-1\} \sum_{\substack{1 \leqq k \leqq n \\
1 \leqq i \leqq r}} \frac{1}{1+x_{k} z_{l}}-\sum_{\substack{1 \leqq l \leqq r \\
1 \leqq k_{1}<\bar{k}_{2} \leqq n}} \frac{2}{\left(1+x_{k_{1}} z_{l}\right)\left(1+x_{k_{2}} z_{i}\right)} \\
& \quad+2 t \sum_{\substack{1 \leqq k \leqq n \\
1 \leqq l_{1}<l_{2} \leqq r}} \frac{1}{\left(1+z_{i_{1}} x_{k}\right)\left(1+z_{i_{2}} x_{k}\right)} \equiv 0 .
\end{aligned}
$$

Proof. Let $d_{z}$ stand for the total derivative with respect to $z=\left(z_{1}, \ldots, z_{r}\right)$. The equality

$$
\begin{aligned}
& d_{z}\left(z_{l}(-)^{i-1} \tilde{\Phi} d z_{1} \ldots d \hat{z}_{i} \ldots d z_{r}\right) \\
& \quad=\left[n+(1-r) t-s-\sum_{1 \leqq k \leqq n} \frac{1}{1+x_{k} z_{i}}+2 t \sum_{\substack{1 \leqq j \leqq r \\
j \neq l}} \frac{z_{i}}{z_{l}-z_{j}}\right] \tilde{\Phi} d \zeta
\end{aligned}
$$

gives

$$
d_{z} \sum_{l=1}^{r}\left((-)^{i-1} z_{i} \tilde{\Phi} d z_{1} \ldots d \hat{z}_{l} \ldots d z_{r}\right)=\left[r(n-s)-\sum_{\substack{1 \leqq k \leqq n \\ 1 \leqq i \leqq r}} \frac{1}{1+x_{k} z_{i}}\right] \tilde{\Phi} d \zeta
$$

since

$$
\sum_{i=1}^{r} \sum_{\substack{1 \leqq j \leqq r \\
j \neq l}} \frac{z_{i}}{z_{i}-z_{j}}=\left(\begin{array}{l}
r \\
2
\end{array}\right) .
$$

It completes the proof of (5).

Subsequently we get

$$
\begin{aligned}
d_{z}( & \left.\frac{z_{l}}{\left(1+x_{k} z_{l}\right)}(-)^{l-1} \tilde{\Phi} d z_{1} \ldots d \hat{z}_{i} \ldots d z_{r}\right)=\left[\frac{(1-r) t-s+n-1}{1+x_{k} z_{i}}\right. \\
& \left.-\sum_{\substack{1<\ell \leqq n \\
\ell \neq \bar{k}}} \frac{1}{\left(1+x_{\ell} z_{i}\right)\left(1+x_{k} z_{l}\right)}+2 t \frac{z_{i}}{1+x_{k} z_{i}} \sum_{\substack{1<j \leqq r \\
j \neq i}} \frac{1}{z_{i}-z_{j}}\right] \tilde{\Phi} d \zeta
\end{aligned}
$$


for $1 \leqq i \leqq r$ and $1 \leqq k \leqq n$, since

$$
\frac{z_{l} x_{l}}{\left(1+x_{\ell} z_{i}\right)\left(1+x_{k} z_{l}\right)}=\frac{1}{1+x_{k} z_{i}}-\frac{1}{\left(1+x_{\ell} z_{i}\right)\left(1+x_{k} z_{l}\right)} \text {. }
$$

Thus we obtain

$$
\begin{aligned}
& d_{z}\left(\sum_{l=1}^{r} \frac{z_{l}}{1+x_{k} z_{i}}(-)^{i-1} \tilde{\Phi} d z_{1} \ldots d \hat{z}_{i} \ldots d z_{r}\right)=\left[\{(1-r) t-s+n-1\} \sum_{1 \leqq l \leqq r} \frac{1}{1+x_{k} z_{i}}\right. \\
& \left.\quad-\sum_{1 \leqq l \leqq r} \sum_{\substack{1 \leqq \ell \leqq n \\
l \neq k}} \frac{1}{\left(1+x_{f} z_{i}\right)\left(1+x_{k} z_{l}\right)}+2 t \sum_{1 \leqq i<j \leqq r} \frac{1}{\left(1+x_{k} z_{i}\right)\left(1+x_{k} z_{j}\right)}\right] \tilde{\Phi} d \zeta .
\end{aligned}
$$

Here we use the identity

$$
\begin{aligned}
& \sum_{i=1}^{r} \sum_{\substack{1 \leqq j \leqq r \\
j \neq l}} \frac{z_{l}}{\left(1+x_{k} z_{i}\right)\left(z_{i}-z_{j}\right)}=\sum_{1 \leqq i<j \leqq r} \frac{z_{i}}{\left(1+x_{k} z_{l}\right)\left(z_{l}-z_{j}\right)} \\
& \quad+\sum_{1 \leqq i<j \leqq r} \frac{z_{j}}{\left(1+x_{k} z_{j}\right)\left(z_{J}-z_{i}\right)}=\sum_{1 \leqq i<j \leqq r} \frac{1}{\left(1+x_{k} z_{l}\right)\left(1+x_{k} z_{J}\right)}
\end{aligned}
$$

Summing up for $k$, we finally obtain

$$
\begin{aligned}
d_{z}\left(\sum_{k=1}^{n} \sum_{l=1}^{r} \frac{z_{i}}{1+x_{k} z_{i}}(-)^{i-1} \tilde{\Phi} d z_{1} \ldots d \hat{z}_{i} \ldots d z_{r}\right) \\
=\left[\{(1-r) t-s+n-1\} \sum_{1 \leqq k \leqq n} \sum_{1 \leqq i \leqq r} \frac{1}{1+x_{k} z_{i}}\right. \\
\quad-\sum_{1 \leqq l \leqq r} \sum_{1 \leqq k<t \leqq n} \frac{2}{\left(1+x_{/} z_{i}\right)\left(1+x_{k} z_{i}\right)} \\
\left.\quad+2 t \sum_{1 \leqq k \leqq n} \sum_{1 \leqq l_{1}<l_{2} \leqq r} \frac{1}{\left(1+x_{k} z_{l_{1}}\right)\left(1+x_{k} z_{i_{2}}\right)}\right] \tilde{\Phi} d \zeta,
\end{aligned}
$$

which leads to the equality (6).

Lemma 5. We have

$$
\begin{gathered}
D(t) \tilde{\Phi}=\left[t\left\{n\left(\begin{array}{l}
r \\
2
\end{array}\right)-(r-1) \sum_{\substack{1 \leqq k \leqq n \\
1 \leqq i \leqq \prime}} \frac{1}{1+x_{k} z_{i}}+\sum_{1 \leqq k \leqq n} \sum_{1 \leqq i<j \leqq r} \frac{1}{\left(1+x_{k} z_{l}\right)\left(1+x_{k} z_{j}\right)}\right\}\right. \\
\left.+r\left(\begin{array}{l}
n \\
2
\end{array}\right)-\sum_{1 \leqq k \leqq r} \sum_{1 \leqq l<J \leqq n} \frac{1}{\left(1+x_{i} z_{k}\right)\left(1+x_{j} z_{k}\right)}\right] \tilde{\Phi} .
\end{gathered}
$$


Proof. Note that

$$
\begin{aligned}
x_{i} \partial_{x_{l}} \tilde{\Phi} & =x_{i}\left\{\frac{z_{1}}{1+x_{i} z_{1}}+\cdots+\frac{z_{r}}{1+x_{i} z_{r}}\right\} \tilde{\Phi} \\
& =\left\{r-\frac{1}{1+x_{i} z_{1}}-\cdots-\frac{1}{1+x_{i} z_{r}}\right\} \tilde{\Phi}
\end{aligned}
$$

and

$$
\begin{aligned}
& x_{i}^{2} \partial_{x_{l}}^{2} \tilde{\Phi}= 2 \sum_{1 \leqq j_{1}<j_{2} \leqq r} \frac{x_{i}^{2} z_{j_{1}} z_{j_{2}}}{\left(1+x_{i} z_{j_{1}}\right)\left(1+x_{l} z_{j_{2}}\right)} \tilde{\Phi} \\
&= 2 \sum_{1 \leqq j_{1}<j_{2} \leqq r}\left(1-\frac{1}{1+x_{i} z_{j_{1}}}\right)\left(1-\frac{1}{1+x_{l} z_{j_{2}}}\right) \tilde{\Phi} \\
&= 2\left[\left(\begin{array}{l}
r \\
2
\end{array}\right)-\sum_{1 \leqq j_{1}<J_{2} \leqq r}\left(\frac{1}{1+x_{l} z_{j_{1}}}+\frac{1}{1+x_{i} z_{j_{2}}}\right)\right. \\
&\left.+\sum_{1 \leqq j_{1}<j_{2} \leqq r} \frac{1}{\left(1+x_{l} z_{j_{1}}\right)\left(1+x_{i} z_{j_{2}}\right)}\right] \tilde{\Phi} \\
&=2 {\left[\left(\begin{array}{l}
r \\
2
\end{array}\right)-(r-1) \sum_{1 \leqq j \leqq r} \frac{1}{1+x_{i} z_{j}}\right.} \\
&\left.\quad+\sum_{1 \leqq j_{1}<j_{2} \leqq r} \frac{1}{\left(1+x_{l} z_{j_{1}}\right)\left(1+x_{i} z_{j_{2}}\right)}\right] \tilde{\Phi} .
\end{aligned}
$$

Hence, the equality

$$
\begin{aligned}
\sum_{1 \leqq j_{1} \neq J_{2} \leqq n} \frac{x_{j_{1}}}{\left(x_{j_{1}}-x_{j_{2}}\right)\left(1-x_{j_{1}} z_{i}\right)}= & \sum_{1 \leqq j_{1}<j_{2} \leqq n} \frac{x_{j_{1}}}{\left(x_{j_{1}}-x_{j_{2}}\right)\left(1-x_{j_{1}} z_{i}\right)} \\
& +\sum_{1 \leqq j_{1}<j_{2} \leqq n} \frac{x_{j_{2}}}{\left(x_{j_{2}}-x_{j_{1}}\right)\left(1-x_{j_{2}} z_{i}\right)} \\
= & \sum_{1 \leqq j_{1}<j_{2} \leqq n} \frac{1}{\left(1+z_{i} x_{j_{1}}\right)\left(1+z_{i} x_{j_{2}}\right)}
\end{aligned}
$$

implies (7).

At this stage, combining Lemmas $4-5$, we reach the desired assertion:

$$
\left[D(t)-e_{\left(r^{s}\right)}(t)\right] \int_{\mathscr{C}} \tilde{\Phi} d \zeta=0
$$




\section{References}

1. Bauer, M., Di Francesco, P., Itzykson, C., Zuber, J.B.: Covariant differential equations and singular vectors in Virasoro representations. Nucl. Phys. B362, 515-562 (1991)

2. Felder, G.: BRST approach to minimal models. Nucl. Phys. B317, 215-236 (1989), Erratum in B324, 548 (1989)

3. Feigin, B.L., Fuchs, D.B.: Representations of the Virasoro algebra. In: Topology, Proceedings. Leningrad 1982. Faddeev, L.D., Mal'cev, A. (eds.), Lecture Notes in Math., Vol. 1060, Springer, 1984

4. Macdonald, I.G.: Symmetric functions and Hall polynomials. 2nd ed. (Oxford Mathematical Monographs) Oxford University Press, 1995

5. Macdonald, I.G.: Commuting differential operators and zonal spherical functions. In: Algebraic Groups, Utrecht 1986, A.M. Cohen et al. (eds.), Lecture Notes in Math., Vol. 1271, Springer, 1987, pp. $189-200$

6. Macdonald, I.G.: A new class of symmetric functions. Publ. IRMA Strasbourg, Seminaire Lotharingien, 1988, pp. 131-171

7. Stanley, R.P.: Some combinatorial properties of Jack symmetric functions. Adv. in Math. 77, 76-115 (1989)

8. Tsuchiya, A., Kanie, Y.: Fock space representations of the Virasoro algebra-Intertwining operators. Publ. RIMS. Kyoto Univ. 22, 259-327 (1986)

9. Wakimoto, M., Yamada, H.: The Fock representations of the Virasoro algebra and the Hirota equations of the modified KP hierarchy. Hiroshima Math. J. 16, 427-441 (1986)

Communicated by M. Jimbo 
